\title{
DAST optical damage tolerance enhancement and robust lasing via supramolecular strategy
}

Tian, Tian; Wang, Yunkun; Zhang, Wenjing (Angela); Wang, Bing; Fan, Cheng; You, Guanjun; Yuan, Shuai; Xu, Gongjie; Li, Min; Xu, Chuan

Total number of authors:

11

Published in:

ACS Photonics

Link to article, DOI:

10.1021/acsphotonics.0c00602

Publication date:

2020

Document Version

Peer reviewed version

Link back to DTU Orbit

Citation $(A P A)$ :

Tian, T., Wang, Y., Zhang, W., Wang, B., Fan, C., You, G., Yuan, S., Xu, G., Li, M., Xu, C., \& Cai, B. (2020).

DAST optical damage tolerance enhancement and robust lasing via supramolecular strategy. ACS Photonics, 7(8), 2132-2138. https://doi.org/10.1021/acsphotonics.0c00602

\section{General rights}

Copyright and moral rights for the publications made accessible in the public portal are retained by the authors and/or other copyright owners and it is a condition of accessing publications that users recognise and abide by the legal requirements associated with these rights.

- Users may download and print one copy of any publication from the public portal for the purpose of private study or research.

- You may not further distribute the material or use it for any profit-making activity or commercial gain

- You may freely distribute the URL identifying the publication in the public portal 


\section{Article}

\section{DAST optical damage tolerance enhancement and robust lasing via supramolecular strategy}

Tian Tian, Yunkun Wang, Wenjing Zhang, Bing Wang, Cheng Fan, Guanjun You, Shuai Yuan, Gongjie Xu, Min Li, Chuan Xu, and Bin Cai

ACS Photonics, Just Accepted Manuscript • DOI: 10.1021/acsphotonics.0c00602 • Publication Date (Web): 01 Jul 2020

Downloaded from pubs.acs.org on July 6, 2020

\section{Just Accepted}

"Just Accepted" manuscripts have been peer-reviewed and accepted for publication. They are posted online prior to technical editing, formatting for publication and author proofing. The American Chemical Society provides "Just Accepted" as a service to the research community to expedite the dissemination of scientific material as soon as possible after acceptance. "Just Accepted" manuscripts appear in full in PDF format accompanied by an HTML abstract. "Just Accepted" manuscripts have been fully peer reviewed, but should not be considered the official version of record. They are citable by the Digital Object Identifier (DOI®). "Just Accepted" is an optional service offered to authors. Therefore, the "Just Accepted" Web site may not include all articles that will be published in the journal. After a manuscript is technically edited and formatted, it will be removed from the "Just Accepted" Web site and published as an ASAP article. Note that technical editing may introduce minor changes to the manuscript text and/or graphics which could affect content, and all legal disclaimers and ethical guidelines that apply to the journal pertain. ACS cannot be held responsible for errors or consequences arising from the use of information contained in these "Just Accepted" manuscripts. 


\section{DAST optical damage tolerance enhancement and}

\section{robust lasing via supramolecular strategy}

Tian Tian', Yunkun Wang ${ }^{1}$, Wenjing Zhang ${ }^{2}$, Bing Wang ${ }^{3}$, Cheng Fan' ${ }^{1}$, Guanjun You',

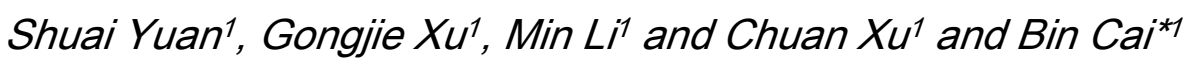

${ }^{1}$ Shanghai Key Lab of Modern Optical System, Ministry of Education, University of Shanghai for Science and Technology, Shanghai 200093, China

${ }^{2}$ Department of Environmental Engineering, Technical University of Denmark (DTU), 2800 Kgs. Lyngby, Denmark.

${ }^{3}$ State Key Laboratory Breeding Base of Coal Science and Technology Co-founded by Shanxi Province and the Ministry of Science and Technology, Taiyuan University of Technology, Taiyuan 030024, China

*bullcai@usst.edu.cn 
ABSTRACT. Crystalline 4-N, N-dimethylamino-4'-N'-methyl-stilbazolium tosylate (DAST) is known as an excellent organic nonlinear optical (NLO) material. However, it is vulnerable to damage caused by high-intensity laser irradiation, which severely limits its NLO applications. In this study, DAST molecules were included with $\beta$-cyclodextrin ( $\beta$ CD), and DAST@ $@-C D$ supramolecular nanowires (NWs), microplates (MPs), and chaotic microcrystals (CMCs) were successfully prepared. All the crystals not only possess excellent NLO properties, but also exhibit greatly enhanced optical damage tolerances (ODTs) and photoluminescence. Under the pumping of a 532-nm picosecond (ps) pulsed laser, we achieved single-mode lasing from MPs and random lasing from CMCs, with thresholds of 55.22 and $88.38 \mathrm{~nJ} / \mathrm{cm}^{2}$, respectively. When the laser irradiation had a peak intensity of $\sim 17.5 \mathrm{GW} / \mathrm{cm}^{2}$, which is $8-9$ orders of magnitude higher than the DAST bulk-crystal photobleaching intensity, the CMC laser emission intensity retained $\sim 95 \%$ of its initial value after $4 \mathrm{~h}$ of operation in an ambient atmosphere.

\footnotetext{
KEYWORDS: DAST@ $\beta-C D$, DAST, supramolecule, nanocrystal, optical damage tolerance, lasing
} 


\section{Introduction}

The great variety of organic molecules means that an abundance of organic optical crystals with excellent properties have been developed. These constitute a very promising type of nanophotonic material that may provide an alternative approach for overcoming the physical limitations of integrated circuits ${ }^{1-3}$. The organic ionic crystal $4-N, N$ dimethylamino-4'- $N$-methyl-stilbazolium tosylate (DAST) is recognized as a benchmark organic nonlinear optical (NLO) material due to its very large second-order NLO susceptibilities, electro-optical (EO) figure of merit, and low dielectric constants ${ }^{4-6}$. Although many potential applications such as second-harmonic generation (SHG), ultrahigh-speed EO modulation, terahertz wave generation, and detection have been expected ${ }^{7-12}$, the practical applications of DAST crystals have thus far been limited because of their low optical damage thresholds (ODTs). For example, DAST crystals are easily photobleached by blue or green laser light ${ }^{13,14}$, and even upon irradiation by nearinfrared laser light beyond its absorption band, the ODT is still severely affected by crystal defects ${ }^{15}$. Therefore, it is not possible to utilize the DAST crystal as a laser gain medium, despite the fact that the stilbazolium chromophore is an excellent laser dye that can be 
excited by two-, three-, or even four-photon pumping processes, in solution or in a metalorganic framework host ${ }^{16-19}$. Furthermore, to prepare DAST crystals, the growth process needs to be maintained in a strictly controlled non-aqueous environment, because the stilbazolium chromophore loses its non-centrosymmetric arrangement upon hydration ${ }^{5}$, 6.

Host-guest chemistry is an effective approach for controlling bulk dipolar alignment to achieve SHG materials ${ }^{20-22}$. For example, $D-\pi-A$ NLO chromophores can be included in clathrate hosts such as cyclodextrins (CDs) to form non-centrosymmetric supramolecular crystals even from aqueous solutions ${ }^{21}$. In addition, by inclusion, the microenvironment of the chromophores will be greatly altered, and thus strongly enhanced fluorescence can also be expected ${ }^{23}$. Moreover, the $\beta-C D$ supramolecular structure can effectively increase trans-cis photo-isomerization activation energy $(\Delta E)$ by the interaction between the phenyl ring and the rim of the cavity ${ }^{24}$, while the isomerization of stilbazolium chromophore is a necessary step before the decomposition (Figure 1) ${ }^{14}$. Thus, the $\beta-C D$ supramolecular structure could also be a promising way to increase the ODT of the DAST. Herein, we inserted DAST molecules into $\beta$-CD barrels and prepared 
DAST@ $\beta-C D$ supramolecular crystals, including single crystal nanowires (NWs), microplates (MPs), and chaotic microcrystals (CMCs). All the supramolecular crystals not only possess greatly enhanced ODT and fluorescence, but also exhibit excellent secondorder NLO properties. We achieved single-mode lasing from the MPs and random lasing from the CMCs, with pumping thresholds of 55.22 and $88.38 \mathrm{~nJ} / \mathrm{cm}^{2}$, respectively. The CMCs are particularly stable, retaining $95 \%$ of their initial laser emission intensity after 4 $\mathrm{h}$ of exposure to pumped laser irradiation at an intensity ten-fold greater than the lasing excitation threshold intensity, $P_{\mathrm{th}}$, in an ambient atmosphere.

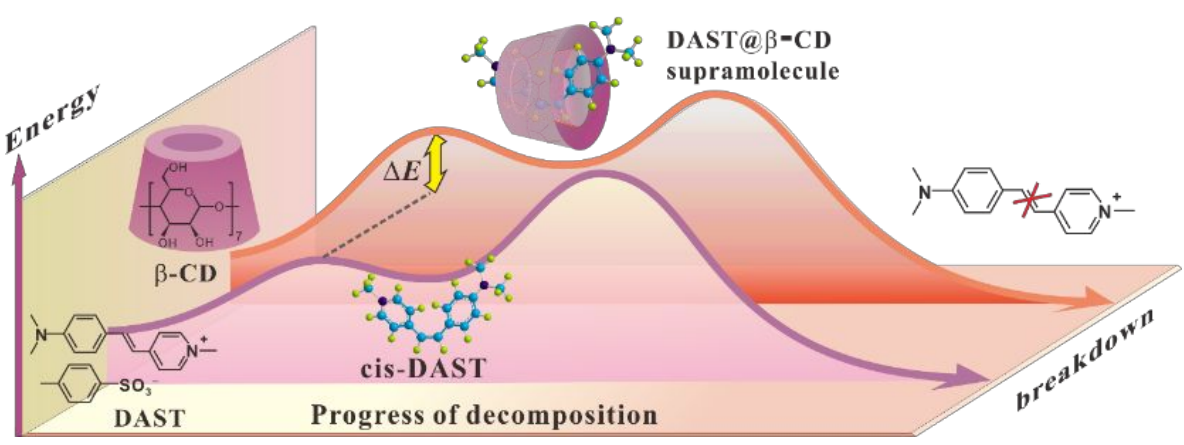


Figure 1. Molecular structures of DAST, $\beta-C D$, and schematic of the decomposition process. By the $\beta-C D$ inclusion the trans-cis isomerization activation energy can be increased $(\Delta E)$.

\section{RESULTS AND DISCUSSION}

DAST@ $\beta-C D$ crystals were fabricated by a surface-supported rapid-evaporation crystallization method and an additional cultivation process ${ }^{25-28}$. More details of this process are given in the Materials and Experimental sections of the Supplementary Information. The morphologies of the DAST@ $\beta-C D N W s$ were studied by scanning electron microscopy (SEM), and the results are shown in Figure 2(a-b). The DAST@ßCD NWs have several hundred micrometers long and the typical width and thickness are $1 \pm 0.5$ and $0.7 \pm 0.2 \mu \mathrm{m}$, respectively. For comparison, an SEM image of a DAST NW is shown in Figure 2(c). Both types of NW crystallized well, having the very smooth surfaces, which are essential for light confinement and propagation, however, the DAST@ $\beta-C D$ NW image displays a different crystal angle to that of the DAST NW. The X-ray diffraction (XRD) measurements were carried out on the DAST@ $\beta$-CD NWs (Figure S1), and these further elucidate the differences between DAST@ $\beta-C D$ NWs and DAST NWs. As for the XRD of DAST@ $\beta-C D C M C s$, due to the $\beta-C D$ is overdosed, which resulted in $\beta-C D$ dominate the crystallization, thus only the peak from the $\beta$-CD crystal can be detected. The ${ }^{1} \mathrm{H}$ nuclear magnetic resonance $\left({ }^{1} \mathrm{H}\right.$ NMR) spectra of DAST, $\beta-C D$, and DAST@ $\beta$ CD shown in Figure S2, according to the results, we deduced that the DAST molecule enters the $\beta$-CD with preferential insertion of the dimethylaminostyryl group and remains the positively charged pyridinium moiety and tosylate anion out of the $\beta$-CD barrel. Due to the the electrostatic field of the ions, the $\beta$-CD exterior protons have larger shifts than the interior protons. On the other hand, the interaction between the $\beta-C D$ and included DAST moiety is Van der Waals force, which is far weaker than the Colombian force, thus the shifts are almost neglectable. $\pi$ conjugated bond of DAST priority enters $\beta-C D$ molecule cavity. Accordingly, it expands $\beta-C D$ molecule's diameter to form the DAST @ $\beta$-CD inclusion complex. The DAST@ $\beta$-CD NW has a perfect crystal surface with a fringe distance of $4.7 \AA$, as observed by highresolution transmission electron microscopy (HRTEM; Figure 2(c)). The selected-area electron-diffraction (SAED) pattern is shown in Figure 2(d). The regular array of diffraction spots indicates the single crystalline nature of the DAST@ß-CD NW. 


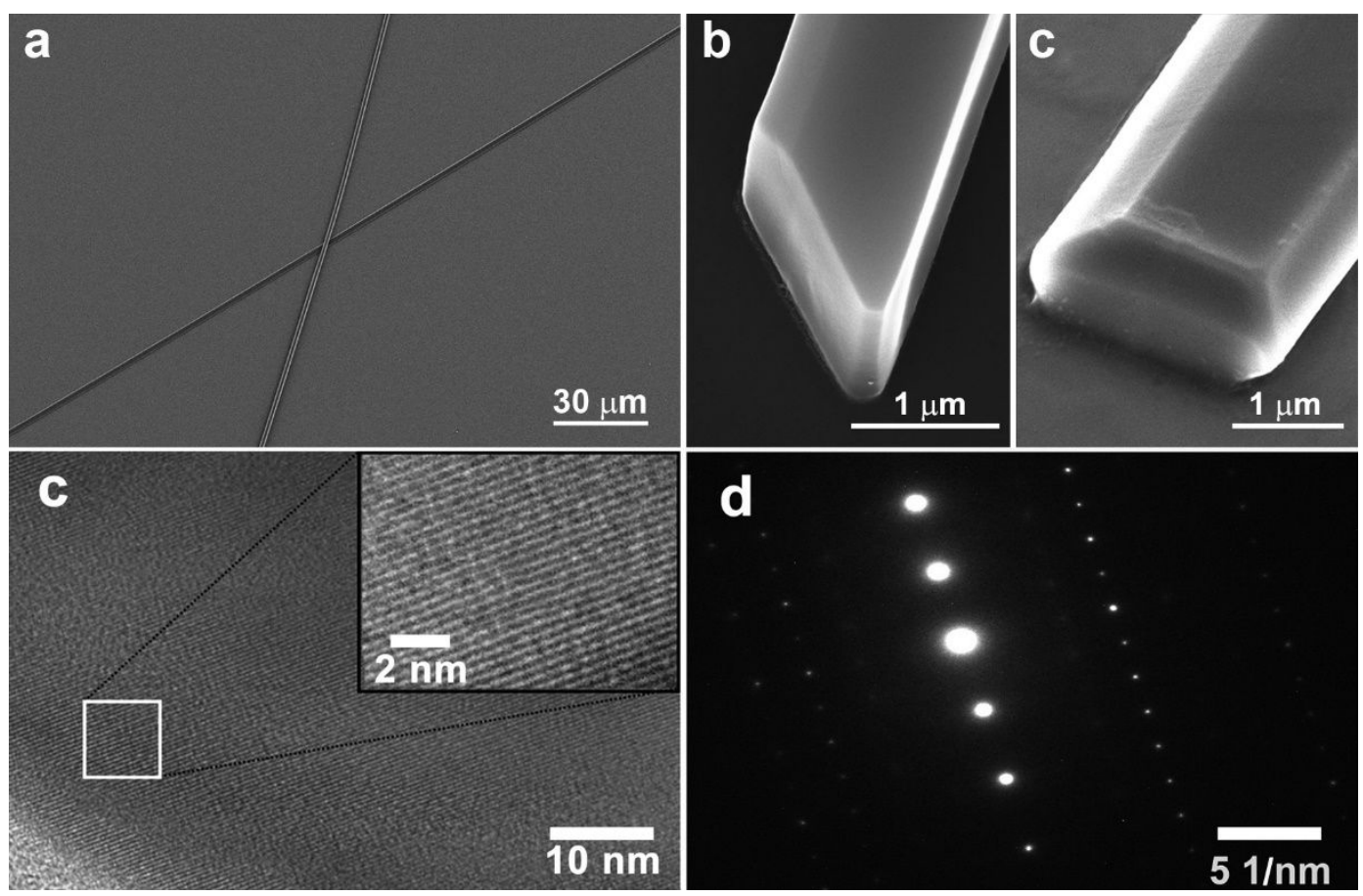

Figure 2. Morphology of DAST@ $\beta-C D$ NW. (a-b) SEM images of DAST@ $\beta-C D$ NW. (c)

SEM image of DAST NW. (c) HRTEM image of DAST@ $\beta-C D N W$; the inset shows a partial enlarged view. (d) DAST@ $\beta-C D$ NW SAED pattern.

By its inclusion within $\beta-C D$, the PL properties of the DAST molecule can be dramatically enhanced. The PL spectra of pure $\beta-C D$, pure DAST, and DAST@ $\beta-C D$ water solutions with different molar ratios (DAST: $\beta-C D=1: 2 ; 1: 4$; and $1: 6$ ) are shown in Figure $3(a)$. We can see that as the proportion of the $\beta-C D$ increases, $\sim 10$-fold higher fluorescence quantum yields can be achieved by the DAST@ $\beta-C D$ supramolecular structure. 

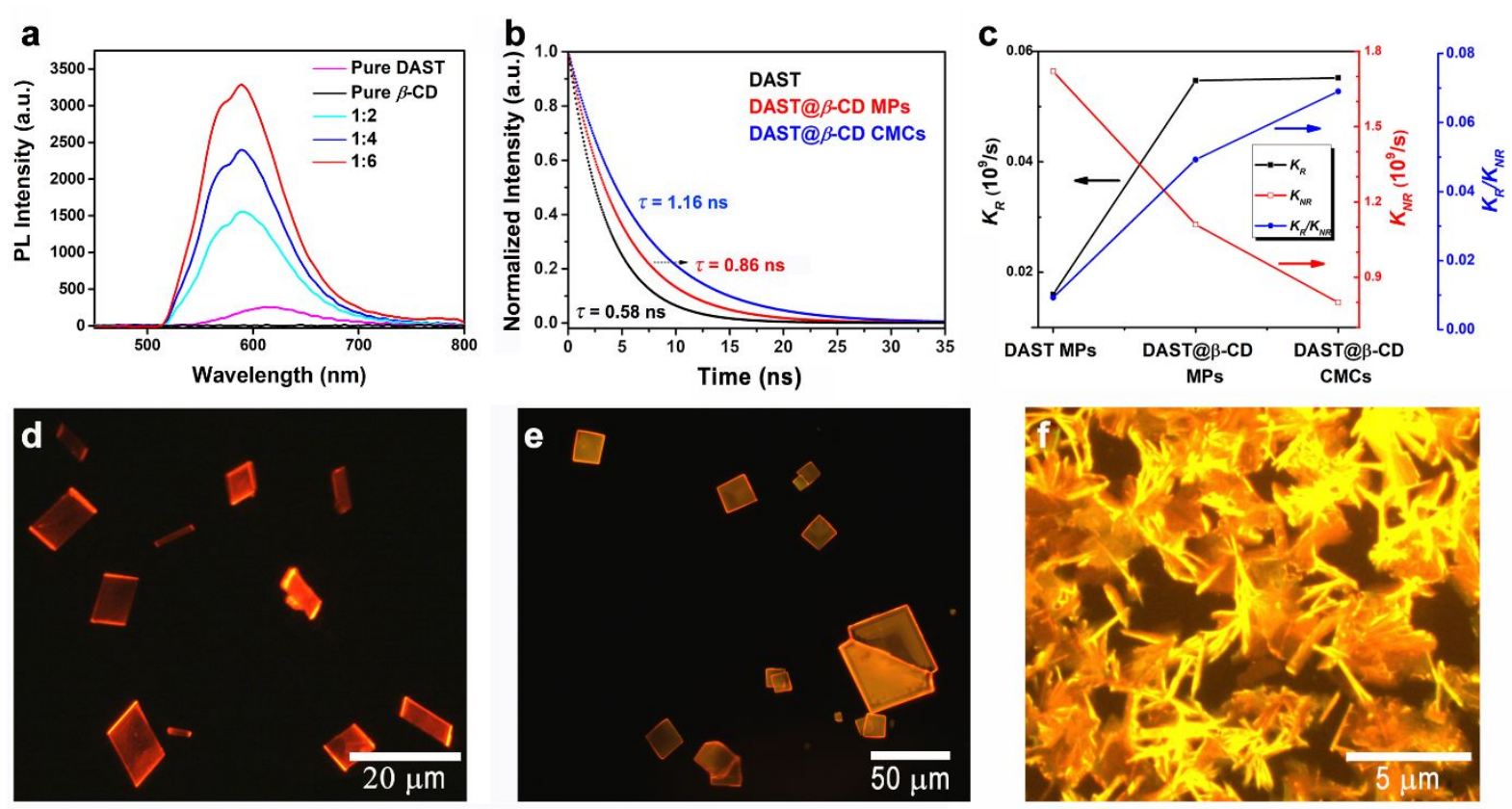

Figure 3. PL properties of DAST@ $\beta-C D$ samples. (a) PL spectra of pure $\beta-C D$, DAST, and $\beta$-CD@DAST solutions with different DAST: $\beta-C D$ molar ratios. (b) Fluorescence lifetimes of $\beta$-CD@DAST MPs, $\beta-C D @ D A S T ~ C M C s$, and DAST MPs. (c) Radiative and nonradiative transition rates of DAST MPs, DAST@ $\beta-C D$ MPs, and DAST@ $\beta-C D C M C s$. (d-f) PL images of DAST@ $\beta-C D$ MPs, DAST MPs, and DAST@ $\beta-C D ~ C M C s$.

The PL lifetime can also be enhanced by the inclusion of the fluorophore in the supramolecular structure. The time-resolved fluorescence decay data for the DAST@ßCD MPs, DAST@ $\beta-C D$ CMCs, and DAST NCs are shown in Figure 3(b). The fluorescence lifetime $T$ was calculated by fitting the curves using a multi-exponential 
model. The lifetime of the DAST MPs is 0.58 ns, but the lifetimes of the DAST@ $\beta-C D$ MPs and DAST@ß-CD CMCs are increased to 0.86 and 1.16 ns, respectively. An appropriately long lifetime is beneficial for population inversion in the lasing process. The photoluminescence quantum yields (PLQY) of the DAST MPs, DAST@ $\beta-C D$ MPs, and DAST@ $\beta$-CD CMCs are evaluated as $0.9 \%, 4.5 \%$ and $6.8 \%$, respectively (Hamamatsu Quantaurus-QY, Figure S3). The radiative transition rate $\left(K_{R}\right)$ and nonradiative transition rate $\left(K_{N R}\right)$ can be given by the Equation 1 and $2{ }^{29}$.

$$
\begin{gathered}
K_{R}=\Phi / \tau \\
K_{N R}=K_{R} / \Phi-K_{R}
\end{gathered}
$$

where $\Phi$ is the PLQY. From the calculation results shown in Figure 3(c), we can see that the DAST@ $\beta-C D$ supramolecular structure will efficiently increase the $K_{R}$ and decrease the $K_{N R}$ of DAST. After the $\beta-C D$ inclusion, the $K_{R} / K_{N R}$ increased from 0.009 to 0.049 and

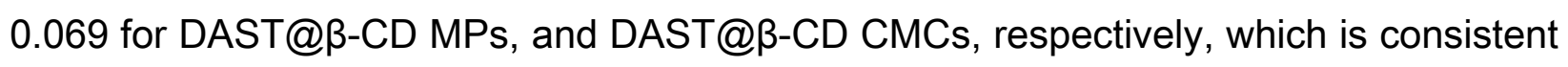
well with the PL spectral results. 
Photoluminescence (PL) photographs of the DAST@ $\beta-C D$ MPs and DAST MPs are shown in Figure 3(d) and (e). The DAST@ $\beta-C D$ MPs have a rhomboid shape, and the short edges are always brighter than the long edges, regardless of their orientations, which strongly suggests their optical anisotropy; however, we cannot find similar phenomenon from the DAST MPs. A PL photograph of some typical CMCs is shown in Figure 3(f); these particles are random and without particular shapes. Under the same excitation conditions, the CMCs are obviously brighter than the MPs, which is also consistent well with its highest $K_{R} / K_{N R}$ values.

The second-order NLO properties of DAST@ß-CD MPs, NWs, and CMCs were investigated by performing SHG measurements using the setup illustrated in Figure S4. A femtosecond (fs) pulsed laser (1030 nm, repetition rate $300 \mathrm{kHz}$, pulse duration $800 \mathrm{fs}$ ) was utilized as the pumping source. DAST@ $\beta-C D$ NW and MP SHG images are shown as insets on the left of Figure 4(a), where we can see that the entire surfaces of the DAST@ $\beta-C D ~ N W$ and MP emit green light, despite the strong absorptions in this spectral region. The SHG signal of the DAST@ $\beta-C D$ MP at $515 \mathrm{~nm}$, corresponding to the 
frequency-doubled $1030-\mathrm{nm}$ pump laser signal, is sharp and intense, indicating that the very high second-order nonlinearity of the DAST crystal is retained. We monitored the SHG responses by varying the polarization of the incident laser. A polar plot of the SHG signals is shown as the right inset of Figure 4(a). The observed SHG signals have a fourlobe pattern, which is very similar to that produced by DAST NCs ${ }^{30}$. For the DAST@ $\beta-$ CD CMCs, under the same pump fluence $\left(25.5 \mathrm{~mJ} / \mathrm{cm}^{2}\right)$, we not only observed a strong SHG signal but also obvious TPEF as well. The TPEF emission is confirmed by spectroscopy as well as micrograph (left inset, Figure 4(b)), although its intensity is about 1 order of magnitude less than that of the SHG signal. The strongly scattering nature of the CMCs facilitates TPEF observation, while in the cases of the NWs and MPs, the TPEF is strongly confined to the crystals due to their regular shapes and smooth surfaces. Owing to its random nature, the polarization of SHG for CMCs is less sensitive to the incident angle of the pump laser, as illustrated in the polar plot shown as the right inset of Figure 4(b). 

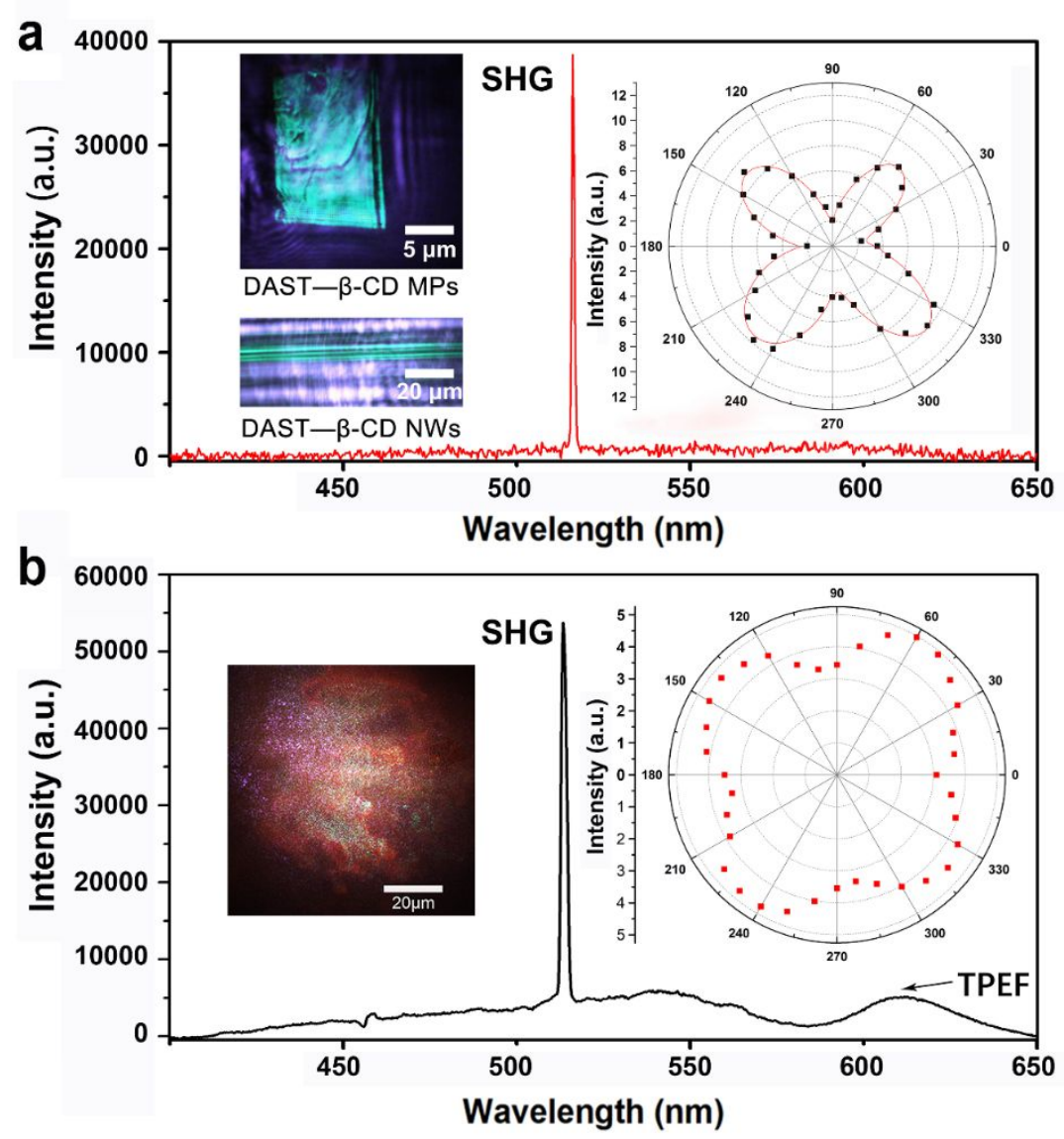

Figure 4. (a) SHG spectrum of DAST@ $\beta-C D$ MPs with SHG images of DAST@ $\beta-C D N W$ and MP (inset left) and polar plot of SHG intensity as a function of incident laser polarization angle (inset right). (b) SHG spectrum of DAST@ $\beta-C D C M C s$ with SHG image of CMCs (inset left) and polar plot of SHG intensity as a function of incident laser polarization angle (inset right).

Because of the greatly enhanced PL intensity and elongated lifetime generated by the supramolecular structure, DAST@ $\beta-C D$ lasing is expected. The MPs and CMCs were 
pumped by a picosecond (ps) pulsed laser $(532 \mathrm{~nm}$, repetition rate $100 \mathrm{~Hz}$, pulse duration 10 ps), within the optical setup shown in Figure S5. In the case of the DAST@ $\beta-C D$ MPs, the smooth facets of the crystals form excellent Fabry-Pérot $(F-P)$ resonant cavities, and thus light amplification is facilitated. The PL intensity as a function of excitation fluence is shown in Figure 5(a). As the excitation energy density increases, a very sharp peak appears within the spontaneous emission spectral range. It is inferred that upon further increasing the excitation intensity, stimulated emission becomes predominant, and lasing modes are excited. The shining ends of the crystal in the inset of Figure 5(a) confirm this point. The laser cavity quality factor $Q$ can be defined as $Q=\lambda / \Delta \lambda$, where $\lambda$ is the wavelength of the spectral peak and $\Delta \lambda$ is its FWHM. The $Q$ factor of this single-mode lasing can be calculated as 937 , using $581 \mathrm{~nm}$ as the peak center and an approximate value of $0.62 \mathrm{~nm}$ for $\Delta \lambda$. Here, we should note that the FWHM measurement and hence also the $Q$ factor estimation are limited by the low resolution $(\approx 0.23 \mathrm{~nm})$ of our fiber spectrometer. The relationship between the PL output and excitation intensities in Figure 5(b) depicts the evolution from spontaneous emission to laser excitation. From the inflection point, a lasing threshold of $55.22 \mathrm{~nJ} / \mathrm{cm}^{2}$ can be obtained; this value is 
considerably low compared with those of other organic laser materials ${ }^{3}$. Lasing stability was evaluated at room temperature in an open environment. After continuously pumping with a 532-nm laser, using an excitation intensity of $4.3 \times P_{\text {th }}$ for $3 \mathrm{~h}$, the laser intensity remains at $\sim 62 \%$ of its initial value. We also attempted to excite DAST MPs because they also can form a perfect F-P cavity under UV irradiation (Figure S6). However, this sample was rapidly ablated under ps pulsed laser irradiation (Figure S7). We speculate that there are at least two reasons for the dramatic ODT improvement for DAST@ $\beta-C D$. First, the guest-host inclusion structure limits the trans-cis isomerization of stilbazolium, the first step in the decomposition of the DAST molecule (Figure 1(a)) ${ }^{13}$. Second, the guest-host system greatly enhances DAST fluorescence, as the energy absorbed from the pump is more efficiently released in the form of photons. As we depicted in Figure 3(c) the $K_{R} / K_{N R}$ can be increased 5-7 folds in the supramolecular structures with respect to that in the DAST crystal, in other words, the nonradiative relaxation can be significantly suppressed. In the case of the DAST@ $\beta-C D$ CMCs, the PL light can be strongly scattered, and gain occurs among the microcrystals, leading to random lasing ${ }^{31-33}$. As shown in Figure 
5(d), at low excitation intensities, the PL spectrum consists of two broad spontaneous emission peaks (yellow line). As the pump power increases, the emission peak becomes narrower because of preferential amplification at frequencies close to the maximum of the gain spectrum (green line). When the excitation intensity exceeds the lasing threshold $\left(P_{\mathrm{th}}=88.38 \mathrm{~nJ} / \mathrm{cm}^{2}\right.$, as obtained from the plot in Figure 5(e)), multiple peaks in the range of 595-610 nm can be observed. When the pump intensity increases further, more modes are excited (blue line). Because of the random nature of the lasing, the frequencies of the CMC laser emission peaks depend on the sample position and the angle of the incident light. In other words, when we change the location or angle of the pump light, the lasing peak wavelengths will also change (Figure S8). The CMCs possess an extraordinary ODT compared to that of the MPs. As shown in Figure 5(f), we continuously irradiated the CMCs with an intensity of $10.1 \times P_{\text {th }}$ for $4 \mathrm{~h}$ in an ambient atmosphere. At the end of this period, the laser signal intensity still retained $\sim 95 \%$ of its initial value. Rather than the MP laser in which the laser cavity is the crystal itself, the lasing of the CMCs results from multiple scattering events between the CMCs, outside the individual crystals. Therefore, much less PL reabsorption occurs in the CMCs than in MPs, and as a result, the CMCs 
can tolerate an irradiation levels of $\sim 17.5 \mathrm{GW} / \mathrm{cm}^{2}$ without breakdown occurring; this value is 8-9 orders of magnitude higher than the intensity at which photobleaching occurs under 532-nm irradiation in the DAST bulk crystal ${ }^{13}$.
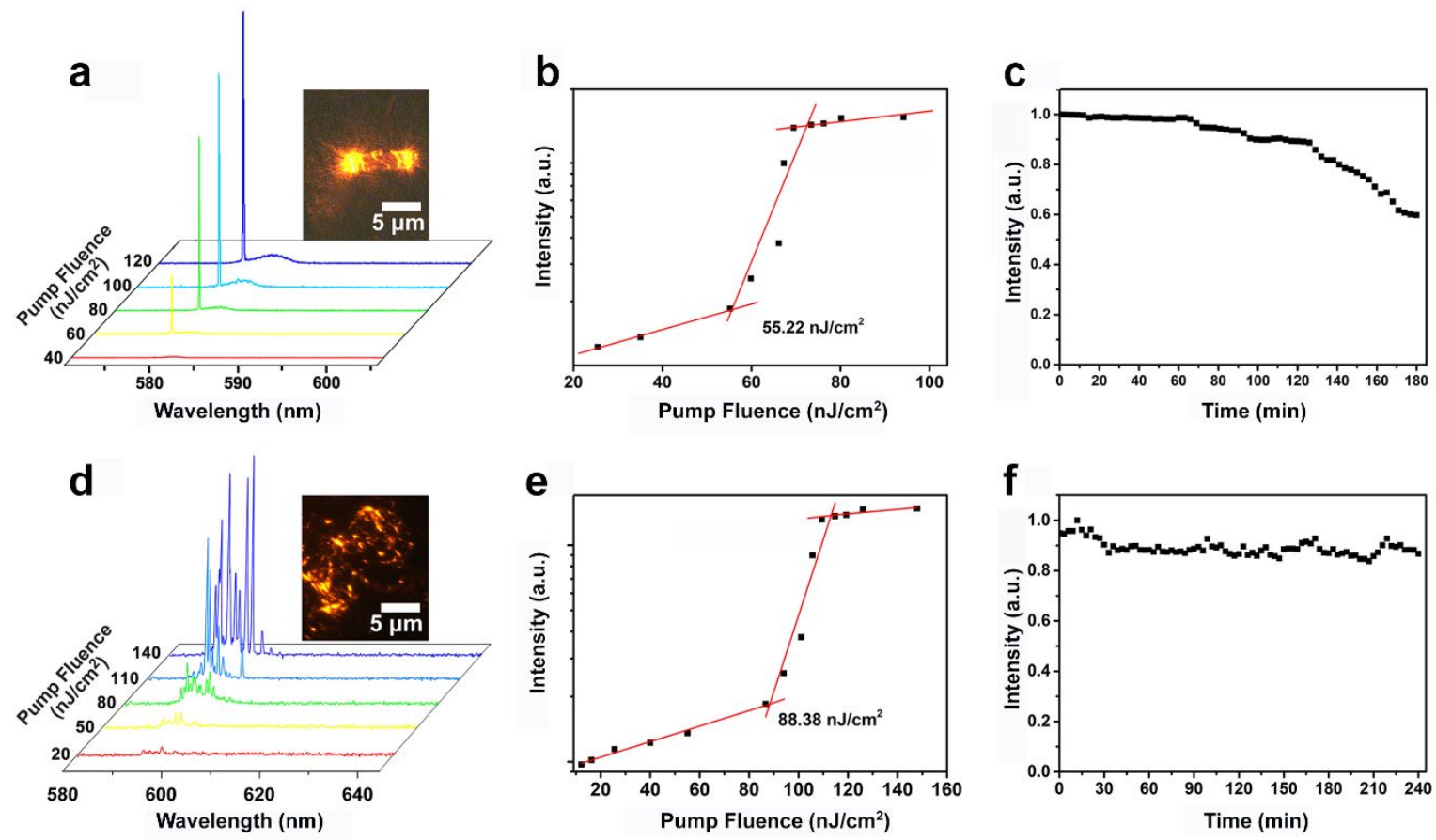

Figure 5. Lasing performances of DAST@ $\beta-C D$ MPs and CMCs. (a) Power-dependent PL spectra of DAST MPs with image of MP lasing (inset). (b) Relationship between output intensity and pump fluence. (c) MP lasing stability under laser irradiation at $4.3 \times P_{\text {th }}$ in an ambient atmosphere. (d) Power-dependent PL spectra of DAST@ $\beta-C D C M C s$ with image of CMC lasing (inset). (e) Relationship between output intensity and pump fluence. (f) DAST@ $\beta-C D$ CMC lasing stability under pump-laser irradiation at $10.1 \times P_{\text {th }}$. 


\section{CONCLUSION}

In this study, a new type of supramolecular optical crystal, DAST@ $\beta-C D$, was successfully prepared by the strategy of host-guest complexation. The DAST@ $\beta-C D$ crystals (CMCs, NWs, and MPs) display several advantageous optical features, including excellent NLO (SHG and TPEF) and PL properties, as well as greatly enhanced ODTs. These features enable the DAST@ $\beta-C D$ crystals to generate stimulated emission upon excitation with a ps pulsed laser. The lasing can be obtained not only by Fabry-Perot resonance, but also via multiple scattering events (random laser), with low excitation thresholds of 55.22 and $88.38 \mathrm{~nJ} / \mathrm{cm}^{2}$, respectively. More importantly, using the inclusion approach, the crystals can possess 8-9 orders of magnitude of ODT enhancement with respect to the DAST bulk crystal. This feature allows high-intensity laser irradiation of the crystal, and consequently high-power, highly stable SHG or terahertz generation can be realized. The supramolecular inclusion strategy, which combines improved photostability, non-centrosymmetric molecular alignment, and $\mathrm{PL}$, should open a new perspective for the development of high-performance and multi-functional photonic materials in the near future. 


\title{
CORRESPONDING AUTHOR
}

\author{
Bin Cai, bullcai@usst.edu.cn
}

\section{ACKNOWLEDGMENTS}

This work was partially supported by the National Key Research and Development

Program of China (No. 2018YFE0205501) and the National Natural Science Foundation

of China, Grants No. 61377111. The authors thank Dr. Jing Chen for assistance with the TEM observation; Mr. Jiyu Yan for assistance with the sample's preparation; Hamamatsu Photonics for photoluminescence quantum yields testing.

\section{SUPPORTING INFORMATION}

Details of the XRD, ${ }^{1} \mathrm{H} N M R, S H G$, and lasing experiments are available as supporting information. Furthermore, any additional relevant information may be obtained via correspondence with the corresponding author.

\section{REFERENCES}


1. Gierschner, J.; Varghese, S.; Park, S. Y. Lasing: Organic Single Crystal Lasers: A Materials View. Adv. Optical. Mater. 2016, 4, 347-347.

2. Zhang, C.; Yan, Y.; Zhao, Y. S.; Yao, J. N. From Molecular Design and Materials Construction to Organic Nanophotonic Devices. Acc. Chem. Res. 2014, 47, 34483458.

3. Kuehne, A. J. C.; Gather, M. C. Organic Lasers: Recent Developments on Materials, Device Geometries, and Fabrication Techniques. Chem. Rev. 2016, 116, 1282312864.

4. Marder, S. R.; Perry, J. W.; Schaefer, W. P. Synthesis of Organic Salts with Large Second-order Optical Nonlinearities. Science. 1989, 245, 626-628.

5. Marder, S. R.; Perry J. W.; Yakymyshyn, C. P. Organic Salts with Large Secondorder Optical Nonlinearities. Chem. Mater. 1994, 6, 1137-1147. 
6. Pan, F.; Wong, M. S.; Bosshard, C.; Peter, G. Crystal Growth and Characterization of the Organic Salt 4-N, N-dimethylamino-4'-N'-methyl-stilbazolium tosylate (DAST). Adv. Mater. 2004, 8, 592-595.

7. Jazbinsek, M.; Mutter, L.; Gunter, P. Photonic Applications with the Organic Nonlinear Optical Crystal DAST. IEEE J. Sel. Top. Qutant. 2008, 14, 1298-1311.

8. Fan, S. Z.; Qi, F.; Notake, T.; Nawata, K.; Matsukawa, T.; Takida, Y.; Minamide, H. Diffraction-limited Real-time Terahertz Imaging by Optical Frequency UpConversion in a DAST crystal. Appl. Phys. Lett. 2014, 104,101106.

9. Thakur, M.; Xu, J.; Bhowmik, A.; Zhou, L. Single-pass Thin-film Electro-Optic Modulator Based on an Organic Molecular Salt. Appl. Phys. Lett.1999, 74, 635-637.

10. Kaino, T.; Cai, B.; Takayama, K. Fabrication of DAST Channel Optical Waveguide. Adv. Funct. Mater. 2002, 12, 599-603.

11. Tonouchi, M. Cutting-edge terahertz technology. Nat. Photonics. 2007, 1, 97-105. 
12. Fan, K.; Xu, X. D.; Gu, Y.; Dai, Z. L.; Cheng, X. M.; Zhou, J.; Jiang, Y. D.; Fan, T.; Xu, J. Organic DAST Single Crystal Meta-Cavity Resonances at Terahertz Frequencies, ACS Photonics. 2019, just accepted.

13. Mutter, L.; Mojca, J.; Zgonik, M.; Meier, U.; Peter, G. Photobleaching and Optical Properties of Organic Crystal 4-N, N-dimethylamino-4'-N'-methyl Stilbazolium Tosylate. Jour. Appl. Phys. 2003, 94, 1356-1361.

14. Cai, B.; Hattori, T.; Deng, H. H.; Komatsu, K.; Zawadzki, C.; Keil, N.; Kaino, T. Refractive Index Control and Grating Fabrication of 4'-N,N-dimethylamino-Nmethyl-4-stilbazolium Tosylate Crystal. Jpn. J. Appl. Phys. 2001, 40, 964-966.

15. Takahashi, Y.; Onduka, S.; Brahadeeswaran, S.; Yoshimura, M.; Mori,Y.; Sasaki, T. Development of DAST Crystals with High Damage Tolerance. Opt. Mater. 2007, 30, 116-118.

16. He, G. S.; Tan, L. S.; Zheng, Q. D.; Prasad, P. N. Multiphoton Absorbing Materials: Molecular Designs, Characterizations, and Applications. Chem. Rev. 2008, 108, $1245-1330$. 
17. Ren, Y.; Fang, Q.; Yu, W. T.; Lei, H.; Tian, Y. P.; Jiang, M. H.; Yang, Q. C.; Mak, T. C. W. Synthesis, Structures and Two-photon Pumped Up-Conversion Lasing Properties of Two New Organic Salts. J. Mater. Chem. 2000, 10, 2025-2030

18. He, G. S.; Lin, T. C.; Chung, S. J.; Zheng, Q.; Lu, C.; Cui, Y.; Prasad, P. N. Two-, three-, and Four-photon-pumped Stimulated Cavity less Lasing Properties of Ten Stilbazolium-dyes Solutions. J. Opt. Soc. Am. B, 2005, 22, 2219-2228.

19. Yu, J.; Cui, Y.; Xu, H.; Yang, Y.; Wang, Z.; Chen, B.; Qian, G. Confinement of Pyridinium Hemicyanine Dye within an Anionic Metal-organic Framework for TwoPhoton-Pumped Lasing. Nat. Comm. 2013, 4, 2719.

20. Eaton, D. F.; Anderson, A. G.; Tam, W.; Wang, Y. Control of Bulk Dipolar Alignment Using Guest-host Inclusion Chemistry: New Materials for Second-harmonic Generation. J. Am. Chem. Soc. 1987, 109, 1886-1888.

21. Tomaru, S.; Zembutsu, S.; Kawachi, M.; Kobayashi, M. Second-Harmonic Generation in Inclusion Complexes. J. Inclu. Pheno. Macro. Chem. 1984, 2,885890. 
22. Gu, J.; Yan, Y.; Zhang, C.; Yao, J.; Zhao, Y. S. Inclusion Induced Second Harmonic Generation in Low Dimensional Supramolecular Crystals. J. Mater. Chem. C. 2014, 2, 3199-3203.

23. Grabowski, Z. R.; Rotkiewicz, K.; Rettig, W. Structural Changes Accompanying Intramolecular Electron Transfer: Focus on Twisted Intramolecular Charge-Transfer States and Structures. Chem. Rev. 2003, 103, 3899-4031.

24. Guarino, A.; Possagno, E.; Bassanelli, R. Phototransformations in Nonhomogeneous Media: cis- trans-Isomerization of Olefins in Inclusion Compounds. Tetrahedron 1987, 43, 1541-1549.

25. Tian, T.; Cai, B.; Sugihara, O. DAST Single-Nanometer Crystal Preparation Using a Substrate-Supported Rapid Evaporation Crystallization Method. Nanoscale, 2016, $8,18882-18886$.

26. Guan, S.T.; Wang, X. P.; Li, Y.; Tian, T.; Xu, G. J.; Yuan, S.; Cai, B. One-drop Selfassembly and Size Control of Organic Nonlinear Optical Crystal DSNS Nanowires. Cryst. Growth. Des. 2019, 19, 780-786. 
27. Tian, T; Cai, B.; Cheng Q. Q.; Fan, C.; Wang, Y. Y.; Xu, G. J.; Gu, F. X.; Liao, F.;

Sugihara, O.; Hase, E.; Yasui, T. One-Drop Self-Assembly of Ultra-Fine Second-

Order Organic Nonlinear Optical Crystal Nanowires. Nanoscale Res. Lett. 2019, 14:269.

28. Xu, G. J.; Li, Y.; Yan, J. Y.; Lv, X. D.; Liu, Y.; Cai, B. In-Plane Self-Assembly and Lasing Performance of Cesium Lead Halide Perovskite Nanowires, Mater. Res. Lett. 2019, 7 203-209.

29. Chen, W.W.; Shi, T.C.; Du, J.; Zang, Z.G.; Yao, Z.Q.; Li, M.; Sun, K.; Hu, W.; Leng, Y.X.; Tang, X.S. Highly Stable Silica-Wrapped Mn-Doped $\mathrm{CsPbCl}_{3}$ Quantum Dots for Bright White Light-Emitting Devices. ACS Appl. Mater. Interfaces 2018, 10, $43978-43986$.

30. Zheng, M. L.; Fujita, K.; Chen, W. Q.; Duan, X. M.; and Kawata, S. Two-Photon Excited Fluorescence and Second-Harmonic Generation of the DAST Organic Nanocrystals. J. Phys. Chem. C 2011, 115, 8988-8993. 
31. Cao, H.; Jiang, X.; Ling, Y.; Xu, J. Y.; Soukoulis, C. M. Mode Repulsion and Mode Coupling in Random Lasers. Phys. Rev. B. 2003, 67, 161101-161114.

32. Wiersma D. S. The Physics and Applications of Random Lasers. Nat. Phys. 2008, 4, 359-367.

33. Zhu, H. M.; Fu, Y. P.; Meng, F.; Wu, X. X.; Gong, Z. Z.; Ding, Q.; Gustafssonet, M. V.; Trinhal, M. T.; Jin, S.; Zhu, X. Y. Lead Halide Perovskite Nanowire Lasers with Low Lasing Thresholds and High Quality Factors. Nat Mater. 2015, 14, 636-642. 